C. Aerts, T.R. Bedding, \& J. Christensen-Dalsgaard, eds.

\title{
HR 4074: an Emission-Free NRP Twin of the Be Star $28 \mathrm{CMa}$
}

\section{S. Štefl}

Astronomical Institute, Academy of Sciences, CZ-251 65 Ondřejov, Czech Republic, e-mail: sstefl@sunstel.asu.cas.cz

Th. Rivinius, D. Baade

European Southern Observatory, Garching bei München, Germany

\section{Introduction}

In spite of considerable progress in the investigation of rapid line-profile variations $(l p v)$ of Be stars, at least two models still compete in the literature: the rotational modulation, assuming mostly corotating circumstellar structures (Balona, 2000), and nonradial pulsations (Maintz et al. and Rivinius et al., these proceedings). Attempts to explain the lpv of Be stars were often connected with the proto-typical star $28(\omega)$ CMa (HR $2749=$ HD 56139, B2IV-Ve, $v \sin i=80$ $\mathrm{km} / \mathrm{s}$ ). Stefl et al. (1998) described variability in three line-profile components: in the narrow component of the line core, in the line core itself and in the wings. The Balmer emission of $28 \mathrm{CMa}$ is variable and has always been observed to be at least moderate, mostly strong.

HR 4074 (HD 89890, B3 III, $v \sin i=70 \mathrm{~km} / \mathrm{s}$ ) has a different history of its emission activity. From a single spectrogram taken in 1893, Pickering (1898) reported an emission in $\mathrm{H} \beta$. Subsequent observations never confirmed this. If it was not mis-identified by Pickering, HR 4074 would be in the probably longest B-star phase known of any Be star. HR 4074 is very probably not an SPB star the spectral type is too early, the rotational velocity too high and light variations too low for this classification. Baade (1984) detected rapid $l p v$ with a period $\mathrm{P} \approx 2.25 \mathrm{~d}$. Our time series analysis of radial velocity $(\mathrm{RV})$ variations, measured as line modes, and that of full profiles give $P=(2.3179 \pm 0.0008) \mathrm{d}$.

The difference in the emission characteristics provides a critical observational test of the rôle of circumstellar structures in the lpv. New spectra ( 46 of HR 4074 and 97 of $28 \mathrm{CMa}$ ) were obtained with the FEROs spectrograph $(\mathrm{R}=48000,3650-9200 \AA)$ at the ESO La Silla Observatory in 1999-2000.

\section{Results and conclusions}

We can list the following commonalities between $28 \mathrm{CMa}$ and HR 4074:

- Both stars have low $v \sin i$ and are very similar in spectral type. Their RV variations, measured as modes, can be fitted by one period, which is stable and coherent on a time scale of at least years. The corresponding amplitudes are different for different spectral lines, but well correlated in both stars. 
- The variations of the narrow core, the core itself, and the line wings are the same. The power distribution shows two main peaks and, beyond the photospheric $v \sin i$, a secondary maximum in both wings. The phase propagation is prograde in the observer's frame.

- In phases of extreme asymmetry, very sharp features ("spikes") appear at about $\mathrm{V}_{\text {syst }} \pm 0.7 v \sin i$.

The BRUCE and KYLIE codes (Townsend, 1998) were used for the lpv modelling. Details of the modelling, optimization of stellar and pulsational parameters and their errors can be found in Rivinius et al. (2001) and Maintz et al. (these proceedings). The best preliminary fit for HR $4074 l p v$ was achieved with the following stellar parameters:

$\begin{array}{llll}M=7.0 \mathrm{M}_{\odot} & T_{\text {eff }}=15100 \mathrm{~K} & V_{\mathrm{eq}}=352 \mathrm{~km} / \mathrm{s} & R_{\mathrm{eq}}=7.94 \mathrm{R}_{\odot} \\ \log g_{\mathrm{eq}}=2.90 & i=13^{\circ} & V_{\mathrm{eq}} / V_{\text {crit }}=0.90 & R_{\mathrm{pol}}=5.80 \mathrm{R}_{\odot} \\ \text { and the pulsation characteristics: } & & \\ \ell=m=+2 & V_{\max }=45 \mathrm{~km} / \mathrm{s} & P_{\text {corot }}=-18.17 \mathrm{~h} & k=1.53\end{array}$

Modeling of the $l p v$ as $n r p$ leads to acceptable basic parameters of the underlying stars. The $l p v$ of both stars is very satisfactorily reproduced in many details by a quadrupole $g$-mode with $\ell=m=+2$. The line-to-line differences in amplitudes of the RV variations are well reproduced by the modeled profiles. A more detailed modeling is needed to verify that the above parameters represent the global and not a local optimum.

The commonalities of the $l p v$ are so pronounced that any viable model must be able to explain both stars with a minimum of differing free parameters. The continuous presence of line emission in $28 \mathrm{CMa}$ but its century long absence from HR 4074 firmly exclude any circumstellar constituent of such a model.

It has been conjectured (Baade, 1987) that Be stars might be recognizeable by their lpv only. The case of HR 4074 would indeed support the idea that (early-type) Be stars in long-term hibernation can be identified in this way.

Acknowledgments. This work was partly supported by the Grant Agency of the Academy of Sciences of the Czech Repblic (AA3003001).

\section{References}

Baade, D. 1984, A\&A, 134, 105

Baade, D. 1987, in Physics of Be stars, eds. A. Slettebak \& T. Snow, (Cambridge University Press), 361

Balona, L.A. 2000, in ASP Conf. Ser., Vol. 214, The Be Phenomenon in EarlyType Stars, eds. M. Smith, H. Henrichs, \& J. Fabregat, 1

Pickering, E.C. 1898, Harvard Circ. No. 224

Rivinius. Th., Baade, D., Štefl, S., et al. 2001, A\&A, 369, 1058

Townsend, R.H.D. 1998, MNRAS, 284, 839 